\title{
Competencias de cultura colaborativa y liderazgo en estudiantes agropecuarios de primer ciclo de carrera universitaria
}

Competences of collaborative culture and leadership in agricultural students of first university career cycle

John E. Franco-Rodríguez ${ }^{* *}$

Ema N. Moreno-Veloz ${ }^{* * *}$

Eufrasio Pérez Navío****

María Castañar Medina Domínguez ${ }^{* * * *}$

Elba Bermúdez Reyes ${ }^{* * * * * *}$

\section{Resumen}

La formación profesional del Ingeniero Agropecuario de la Universidad Católica de Santiago de Guayaquil-Ecuador, ha

\footnotetext{
* Artículo original derivado del Proyecto "Competencias docentes universitarios" Financiado por la Universidad Católica Santiago de Guayaquil entre agosto 2018 y mayo 2019

** Docente Universitaria. Universidad Católica Santiago de Guayaquil. Guayaquil, Ecuador. E-mail: john.franco@cu.ucsg.edu.ec._ ORCID: http://orcid.org/0000-0002-6285-8200.

*** Docente Universitaria. Universidad Católica Santiago de Guayaquil. Guayaquil, Ecuador. E-mail: ema.moreno@cu.ucsg.edu.ec._ORCID: http://orcid.org/0000-0003-3261-3218 **** Docente Universitaria. Universidad de Jaén. Madrid, España. E-mail: epnavio@ujaen.es. ORCID: http://orcid.org/0000-0001-8688-9602

***** Docente Universitaria. Universidad Católica Santiago de Guayaquil. Guayaquil, Ecuador. E-mail: mcastanar@nebrija.es. ORCID: http://orcid.org/0000-0003-35009913

****** Docente Universitaria. Universidad Católica Santiago de Guayaquil. Guayaquil, Ecuador. E-mail: elba.bermudez@cu.ucsg.edu.ec. ORCID: http://orcid.org/0000-0001-7152-8898
}

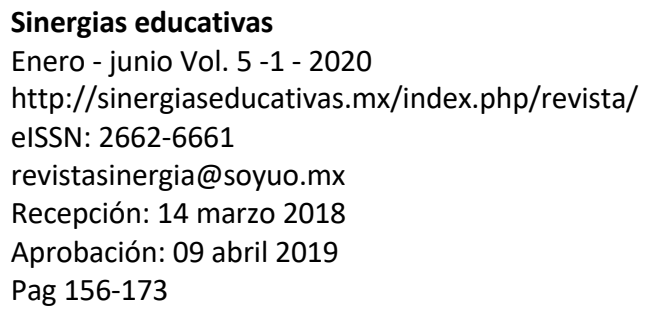

Disponible en

http://www.redalyc.org/articulo.oa?id=57356143200 12

Atribución/Reconocimiento-NoComercialCompartirlgual 4.0 Licencia Pública Internacional CC BY-NC-SA 4.0 https://creativecommons.org/licenses/by-ncsa/4.0/legalcode.es 
transcurrido por diferentes etapas, lo que ha significado que se hayan requerido transformaciones sucesivas a efectos de insertar nuevos modelos para el desarrollo de competencias en el estudiantado y profesorado. El objetivo de esta investigación fue determinar en el estudiantado del primer ciclo de estudios de la carrera de AGROPECUARIA de la FETD-UCSG la valoración que le asigna a las competencias docentes de cultura colaborativa y liderazgo para propiciar la innovación educativa en el aula universitaria. El estudio de enfoque mixto (cualitativo-cuantitativo) de corte transeccional analítico y descriptivo con diseño no experimental. Entre los resultados se puede indicar que los estudiantes definen la importancia de contar con un docente que les sirva de modelo de referencia y acompañamiento en el fomento de la cultura colaborativa y el liderazgo en el aula, lo que les permita la asimilación de habilidades blandas que los potencian para el desempeño profesional. Se evidencia la necesidad de un trabajo armónico entre todos los actores del proceso académico de alta calidad que prepare a los estudiantes para las demandas sociales y laborales del mundo contemporáneo. Es necesario continuar trabajando al interior del colectivo académico de la carrera de Agropecuaria a fin de que se hagan transversales las competencias, en especial una cultura colaborativa que prepare al futuro profesional para enfrentar la diversidad en su desempeño profesional como un líder referente en el contexto del sector agropecuario moderno.

Palabras clave: competencias, cultura colaborativa, liderazgo, agropecuaria.
Abstract
Professional training has gone through different stages, which has meant that successive transformations have been required in order to insert new models for the development of skills in students and teachers. The objective of this research was to determine in the student of the first cycle of studies of the career of AGROPECUARIA of the FETD-UCSG the assessment assigned to the teaching competences of collaborative culture and leadership to promote educational innovation in the university classroom. The mixed approach study (qualitative-quantitative) of analytical and descriptive transectional cut with no design experimental. Among 
the results it can be indicated that the students define the importance of having a teacher that serves as a reference and support model in the promotion of collaborative culture and leadership in the classroom, which allows them to assimilate soft skills that they empower them for professional performance. The need for harmonious work among all actors in the high quality academic process that prepares students for the social and labor demands of the contemporary world is evident. It is necessary to continue working within the academic group of the agricultural career in order to make the competences transversal, especially a collaborative culture that prepares the professional future to face diversity in their professional performance as a leading leader in the context of modern agricultural sector.

Key words: skills, collaborative culture, leadership, agriculture.

\section{Introducción}

La formación profesional de nivel ingenieril en el campo amplio de la Agricultura, Silvicultura, Pesca y Veterinaria en la Facultad de Educación Técnica para el Desarrollo (FETD) de la Universidad Católica de Santiago de Guayaquil (UCSG), ha transcurrido por diferentes etapas, desde la reforma curricular que implementó la carrera de nivel terminal por objetivos procedimentales y actitudinales en 1998, transitando hacia el sistema de créditos académicos en el 2003, yendo hacia un malla por competencias en 2009, seguida de una estructura académica por resultados de aprendizaje en 2012 y en la actualidad, un rediseño curricular por horas que tienen en esencia los escenarios de aprendizaje; en todos los casos emitiendo el título profesional de ingeniero agropecuario.

El sector agropecuario ecuatoriano tiene demandas sociales y laborales que implican que los nuevos profesionales sean capaces de resolver problemas y retos que la globalización y la competitividad deparan a los agroemprendimientos y organizaciones públicas y privadas, por lo que durante su proceso de formación, la academia pone de manifiesto la competencia de cultura colaborativa entre los diferentes colectivos humanos que interactúan en los sistemas agroproductivos con un claro liderazgo en los procesos de valor, que 
direccionen principios corporativos que conduzcan a las instituciones que sean dirigidos por ellos. Lo expuesto se torna en una oportunidad para que la academia evidencie entre su profesorado una declaratoria y puesta en ejecución de las competencias de liderazgo y cultura colaborativa en el colectivo de aula, que genere nuevos profesionales agropecuarios visionarios, emprendedores que sean socialmente responsables.

Con la finalidad de que se afiance y promueva dentro del colectivo docente de las carreras universitarias rediseñadas y en especial, las agropecuarias de la FETD-UCSG, el Vicerrectorado Académico se articula con la Universidad Nacional a Distancia (UNED) de España, para gestionar con el apoyo del Sistema de Investigación y Desarrollo (SINDE) y bajo la ejecución del Centro de Innovación Educativa y Desarrollo Docente (CIEDD), el proyecto "Modelo de mejora de la cultura formativa de los profesores basado en competencias para su desarrollo profesional", en el cual participan profesores que dictan clases en el ciclo básico de la carrera de AGROPECUARIA y del cual se desprende la investigación sobre las competencias trabajadas en conjunto con el colectivo estudiantil sobre el desempeño y aplicación que los profesores ejercen en su praxis rutinaria.

Según Roselli (2016), la cultura colaborativa se puede definir como el conjunto de estrategias direccionadas para que los estudiantes sean actores activos de su propio aprendizaje, es decir que, los grupos estudiantiles evidencien proactividad en el proceso docenteeducativo; es un constructo contemporáneo en las aulas universitarias. Para Cadavieco, Martínez y Cabezas (2016), el trabajo colaborativo es potencialmente innovador entre los estudiantes, ya que los conduce a lograr avances significativos dentro del aprendizaje. El trabajo colaborativo o cooperativo (Torres y Vallejo, 2018) logra un incremento de la participación y responsabilidad del estudiantado para con sus compañeros y consigo mismo; por ello, es clave que el docente universitario cree espacios colaborativos dentro del proceso de impartición de su cátedra, evidenciando con ello la innovación permanente al interior de su praxis profesional. 
El fortalecimiento de la cultura colaborativa, mejora el ambiente de trabajo dentro del colectivo docente, ya que al compartir conocimientos y estrategias de trabajo, así como las formas de relación interpersonal y las posibilidades de asociación para el trabajo generan alta productividad en la comunidad universitaria.

Vinagre (2014), reportó los resultados de un proyecto colaborativo en línea realizado con estudiantes universitarios de español, como lengua extranjera en el Reino Unido y de Inglés en España; utilizaron un sistema informático en sitios web (wiki), el cual permitió a los usuarios inrteractuar con el compañero, esta herramienta rápida $\mathrm{y}$ sencilla resultó una estrategia colaborativa que a la vez permitió mejorar el aspecto intercultural; inclusive para el aprendizaje de lengua extranjera.

La investigación realizada en España (Universidad de Barcelona) por Borrasca (2014), con estudiantes de los grados de Pedagogía y Maestro de Educación Primaria, reporta que la estrategia de seminarios colaborativos es una herramienta que significativamente privilegia el aprendizaje de los estudiantes, a la vez que desarrolla la competencia de trabajo en equipo.

Romero y Garay (2018), reportó en su trabajo investigativo en "Tecnologías de la Información y Comunicación en el Grado Educación Infantil" en la Universidad del País Vasco, el empleo de la red social Facebook, en la cual es crucial la asignación de roles en el grupo y su respectiva rotación de los mismos; lo que corrobora Olivares (2015), según el estudio realizado en la Universidad Nacional Experimental Politécnica de la Fuerza Armada sede Anzoátegui, Venezuela en la materia de Climatología Agrícola se referencia la citada estrategia como destacable en cuanto a que permite el intercambio de saberes, facilita complementariedad de capacidades y mejora el desempeño de relaciones entre individuos.

Según Rodríguez y Espinoza (2017), hay momentos en que se producen limitaciones en el proceso formativo cuando los profesores no logran el dominio de las diversas estrategias colaborativas, sobre todo en los medios virtuales como en el caso de la plataforma digital. 
El trabajo realizado por Paris, Mas y Torrelles (2016), con estudiantes universitarios de las Facultades de Ciencias de la Educación de la Universidad Autónoma de Barcelona y de la Facultad de Educación, Psicología y Trabajo Social de la Universidad de Lleida, dan como resultado que es evidente que la reacción del colectivo estudiantil sobre la competencia de trabajo en equipo puede llegar a anticiparse a las situaciones problemas y de forma estratégica a brindar soluciones alternas, marcando la línea del docente y potenciando su desarrollo (Domínguez, Ruiz y Medina, 2017).

Para Moreno, Chiecher y Paoloni (2015), el desarrollo de la cultura colaborativa con el uso de las redes sociales entre los estudiantes de reciente ingreso a la vida universitaria fomenta el sentido de pertenencia institucional a temprana edad en los alumnos noveles.

Pachano (2014), ha llevado su trabajo de investigación sobre la competencia de cultura colaborativa a nivel de posgrado en Administración de la Educación Básica en la Universidad de Nacional Experimental "Rafael María Baralt (UNERMB), sede Cabimas-Venezuela, encontrando que incluso a éste nivel son aplicables estrategias corporativas y competitivas en los profesionales, lo cual los conduce a lograr objetivos institucionales.

Las asignaturas de orden técnico-práctico son susceptibles de poder trabajarlas desde el abordaje de la competencia colaborativa en asocio con el uso y aplicación de las tecnologías, conforme lo reseñan García, Domínguez y Spitcich (2017), dejando de lado la praxis convencional del docente universitario, imprimiendo innovación en el proceso de aprendizaje.

Para los investigadores Ferrer et al. (2016), al emplear la técnica del aula invertida (flipped classroom) en el Espacio Europeo de Educación Superior en España (EEES) en los grados de Farmacia, Ingeniería Agroalimentaria y Medio Rural e Ingeniería en Biotecnología, la competencia de trabajo colaborativo a nivel multidisciplinar se ve favorecida al igual que el trabajo autónomo y el uso de la comunicación interpersonal con apoyo digital. 
El Liderazgo es una condición personal del individuo con respecto a una situación de su entorno. Medina, Trasfi, Armenteros y Reyna (2015), reportan que la condición de liderazgo está caracterizada por los altos ejecutivos o directivos, los que serían propulsores de un clima laboral adecuado en las instituciones y con ello un reflejo inmediato a la consecución de logros y objetivos predeterminados.

Existen varios modelos de liderazgo, entre ellos el autocrático y el democrático mismos que son opuestos, que fueron analizados por Ávalos, Dávila y López-Walle (2015), en la investigación con deportistas, valorando la relación entre su entrenador y atleta, concluyeron que la confianza en doble vía es decisiva a la hora de conseguir buenos resultados.

Peralta, Gandoy, Jara y Pacenza (2016), han investigado sobre los estilos de liderazgo laissez-faire y transaccional entre estudiantes universitarios en sus prácticas comunitarias, los cuales son los modelos que han recibido mayor empoderamiento por los practicantes.

Según Hernández, Tobón y Vásquez (2015), se ha diseñado el concepto de liderazgo dentro de las organizaciones para el estímulo de los colaboradores y así obtener el logro de las metas, denominado de abordaje socioformativo cuando se hace una aplicación pedagógica, direccionada y de cambio positivo en el caso de equipos directivos y docentes dentro de las instituciones educativas. Los autores aplicaron la cartografía conceptual, permitiendo que los estudiantes sean proactivos en su aprendizaje a partir de la representación gráfica de los contenidos, fomentado lo colaborativo, la metacognición y de forma transversal la ética en su procedimiento.

Es necesario que para que la competencia de liderazgo se pueda traducir en logros significativos, el docente debe recibir la formación complementaria a la técnica, como lo referencian Toledo, Orús y Agudo (2015), en su investigación realizada concluyen en el énfasis que hace el docente en las estrategias y habilidades de liderazgo pedagógico, especialmente de la mujer universitaria conforme lo corrobora Reche, Sachicola y Lucena (2015), lo cual es de suma importancia en las estructuras organizativas. 
Sopo, Salazar, Guzmán y Vera, (2017), en su revisión del estado del arte en materia de liderazgo, concuerdan que dentro de las organizaciones se pondera una similitud entre el denominado liderazgo emprendedor y el transformacional, por lo que CuevasLópez y Díaz-Rosas (2015), indican que la participación de la mujer en dichas acciones de liderazgo, en especial en los cargos directivos más altos es elemento de evolución asertiva.

Ganga-Contreras, Navarrete, Alt y Alarcón (2016), afirman que hay relación entre el estilo de liderazgo del docente y la percepción del estudiantado sobre sus habilidades de líder que ha demostrado.

Escobar, Isabel y Guerra (2017), han estudiado el tema de la percepción del liderazgo en la institución de educación superior con relación a los sistemas de gestión de calidad, encontrando que los miembros de la comunidad universitaria destacan la importancia de que los actores representativos sean los que evidencien liderazgo en la adopción de los cambios estratégicos. Mientras que, Cetzal y Marfileño (2019), destacan que el liderazgo transformacional entre los docentes se encuentra priorizado.

Delgado, Oliver y Rovira (2015), señalan que la competencia de liderazgo en el docente universitario, requiere de una promoción permanente junto con su alumnado, de una planificación estructurada, la puesta en práctica y de una forma de evaluación por resultados.

Magaña-Medina, Aguilar-Morales y Avendaño-Rodríguez (2016), como producto de su investigación detallan que existe una percepción menor por parte de la competencia entre el profesorado conformado por mujeres a diferencia de los hombres, por lo cual es importante seguir investigando dicha situación entre la comunidad universitaria.

Entre los objetivos planteados para la presente investigación se encuentran la percepción y valoración que los estudiantes de la carrera de AGROPECUARIA tienen en base a su profesorado, el cual se encuentra en proceso de innovación en el desempeño de su práctica con relación a las competencias docentes para fomentar y fortalecer la cultura colaborativa y el liderazgo entre el estudiantado. 


\section{Materiales y métodos}

El estudio de enfoque mixto (cualitativo-cuantitativo) (Pérez, Medina y Cachón, 2019) (Medina, Quintero y Valdez, 2013) (Fernández, 2016) (Gómez, 2015) de corte transeccional analítico y descriptivo con diseño no experimental, la población en estudio fue finita y se basó entre los grupos estudiantiles de la carrera de AGROPECUARIA de la FETD-UCSG que recibieron las materias de Orientación Agropecuaria y Química Inorgánica durante los Semestres A-2018 (24 alumnos) y A-2019 (26 alumnos) que estudiaron en el Primer Ciclo, cuyas edades fluctuaron entre los 17 a 22 años y en una relación del $75 \%$ varones y $25 \%$ mujeres. El muestreo ejecutado fue censal y la recogida de opiniones se realizó a través de la técnica de una encuesta empleando un cuestionario semiestructurado de percepciones aplicado por la vía digital (google drive) y física (boleta impresa), diseñado en seis preguntas con la escala Likert y que se verificó su idoneidad por juicio de expertos y con alfa de Cronbach considerado como aceptable. El período de ejecución fue durante cada parcial (antes de las evaluaciones). De la misma forma se auscultó la opinión de los profesores que impartieron las diferentes materias en cada uno de los grupos; los profesores que estuvieron a cargo de las otras asignaturas y su fondo de tiempo de trabajo presencial y autónomo que formaron parte del ciclo fueron seis, a saber: Análisis Matemático (140 horas), Química Inorgánica (105 horas), Biología (105 horas), Humanismo Integral I (105 horas). Lenguaje y Pensamiento (140 horas) y Ofimática para la Educación Superior (105 horas).

Para el procesamiento de datos se empleó la estadística descriptiva a partir del paquete estadístico SPSS (Statistical Package for the Social Sciences) versión gratuita año 2019, con medidas de tendencia central. Se generaron gráficas para facilitar el análisis de la información y la redacción del reporte científico. A manera de reforzar la metodología se procedió a realizar un taller con expertos profesiones de la docencia universitaria que imparten cátedra en la misma institución, así como también, grupos focales con estudiantes escogidos al azar en atención a la diversidad del aula. 


\section{Resultados}

Los estudiantes consultados refieren en un $60 \%$ que sus docentes entre poco a frecuentemente se preocupan de fortalecer la colaboración en el proceso de aprendizaje, mientras que el $30 \%$ restante señala que nunca y casi nunca. Se podría argumentar que dos tercios del profesorado de la carrera de AGROPECUARIA, incentiva las competencias de la cultura colaborativa.

Las respuestas del estudiantado destacan entre ninguna y casi nunca en un $75 \%$ valoran positivamente las actividades promovidas por sus profesores, seguido de un $20 \%$ que respondió entre poco y frecuentemente lo hacen. Esto quiere decir, que los alumnos son sensibles a las estrategias que sus profesores aplican en el aula para fomentar y fortalecer en ellos la cultura colaborativa, por lo que es importante seleccionar de forma adecuada los recursos a emplearse.

Los alumnos participantes de la encuesta de percepción compartieron la información de que nunca y casi nunca en un $75 \%$ el clima en el aula es consecuencia del trabajo que junto a su docente han realizado para el desarrollo de la cultura colaborativa, seguidos de las respuestas de poco y frecuentemente. Con lo encontrado, se puede decir que el profesorado se muestra implicado en la necesidad de una actualización permanente del clima de aula a partir de una mejor y mayor valoración para que sus estudiantes desarrollen todo su potencial para el proceso de aprendizaje.

El $60 \%$ de los alumnos encuestados respondió que nunca y casi nunca sus profesores aplican métodos que ponen en práctica el fomento de la cultura colaborativa, mientras que un $30 \%$ señaló que poco y frecuentemente se trabaja en el aula esta práctica. Con relación a la praxis docente en la Carrera de AGROPECUARIA, se evidencia la necesidad de un acompañamiento asertivo para el desarrollo de la competencia de la cultura colaborativa.

Los alumnos encuestados coincidieron en un $55 \%$ entre casi nunca $\mathrm{y}$ poco sus profesores promueven estrategias didácticas para el trabajo cooperativo o cultura colaborativa para que los procesos de intercambio de comunicación en el aula con sus estudiantes, mientras que un $40 \%$ señala que frecuentemente y casi siempre se 
lo hace. Es evidente que el profesorado de la carrera de AGROPECUARIA se encuentra en proceso de adopción y desarrollo de la promoción de la cultura colaborativa entre sus estudiantes.

Con un $70 \%$ se encontró que los estudiantes valoran entre nunca y casi nunca valoran la competencia de cultura colaborativa para la mejora en su condición en un futuro desempeño profesional; así mismo, se identificó que el $30 \%$ del estudiantado respondió entre poco a casi siempre se encuentra valorado. Se puede argumentar que cerca de las tres cuartas partes de los estudiantes del Primer Ciclo de la carrera de AGROPECUARIA no han percibido significativamente que en su práctica profesional el desarrollo de la competencia de la cultura colaborativa los ayudará en su desempeño futuro.

Luego de realizadas las encuestas entre los estudiantes, se encontró que el $65 \%$ respondió entre poco a casi siempre y un $30 \%$, entre nunca y casi nunca; esta quiere decir que al menos 7 de cada 10 estudiantes de Primer ciclo de la carrera de AGROPECUARIA, coinciden en que su liderazgo ha sido propiciado y fortalecido en el aula de clases de la mano de sus profesores.

Los estudiantes encuestados reseñan que en un $70 \%$ se fomenta el liderazgo de forma responsable como un valor entre los estudiantes entre nunca y poco, mientras que un $30 \%$ respondió entre frecuentemente y siempre. Es evidente que más de las tres cuartas partes del profesorado debe asumir de forma pertinente el desarrollo del liderazgo con sus estudiantes dentro de su praxis rutinaria.

Cuando se les preguntó a los estudiantes del Primer ciclo de la carrera de AGROPECUARIA sobre la evidencia del liderazgo del profesorado en el aula, sus respuesta estuvieron destacando en un 80 $\%$ entre frecuentemente y siempre y un $20 \%$ entre nunca y poco, lo que nos lleva a argumentar que los docentes de dicha oferta académica demuestran de forma clara el liderazgo en el aula con sus alumnos.

La respuesta consignadas por los estudiantes de la carrera de AGROPECUARIA con relación al potenciamiento del liderazgo 
entre ellos por parte de sus profesores con relación a la responsabilidad tanto individual como grupal fueron las siguientes: un $80 \%$ respondieron entre nunca y poco y $20 \%$ entre frecuentemente y casi siempre. Lo encontrado permite argumentar que más de las tres cuartas partes del profesorado debe fortalecer significativamente la competencia del liderazgo entre los estudiantes para que se desempeñen de forma eficiente tanto individual como grupalmente.

Las respuestas de los estudiantes del Primer ciclo de la carrera de AGROPECUARIA, han sido en un $70 \%$ entre poco y casi siempre y un $30 \%$ señaló entre nunca y casi nunca. Esto deja entrever que el liderazgo empieza a fortalecerse entre la comunidad académica del estudiantado llevados de la mano de su profesorado.

El estudiantado del Primer ciclo de la carrera de AGROPECUARIA que participó en la encuesta de percepciones sobre el liderazgo opina que en un $75 \%$ entre frecuentemente y siempre el resto en un $25 \%$ entre nunca y casi nunca; esto quiere decir que más de las tres cuartas partes del alumnado de dicha oferta formativa, identifican a la competencia de liderazgo como un elemento mejorador de sus capacidades como futuro profesional.

\section{Discusión}

Una vez realizado el trabajo de investigación sobre las competencias de Cultura Colaborativa y el Liderazgo de los estudiantes del Primer Ciclo de la Carrera de AGROPECUARIA de la Facultad de Educación Técnica para el Desarrollo de la Universidad Católica de Santiago de Guayaquil, se estable la siguiente discusión:

Los sucesivos cambios y ajustes a través del tiempo en al diseño curricular de la carrera de AGROPECUARIA de la UCSG, han generado la necesidad de contemporizar la inserción y desarrollo de la cultura colaborativa y el liderazgo en los estudiantes que la cursan, propiciando en las instancias de gestión administrativa académica una preocupación permanente por el afianzamiento de dichas competencias (Hernández, Tobón y Vásquez, 2015), conforme lo indica Roselli (2016), lo que brinda a los estudiantes el alcance de 
logros significativos, afincando en ellos el sentido de la responsabilidad (Torres y Vallejo, 2018).

Sobre la base de las respuesta captadas entre los alumnos en la encuesta se deja entrever que el estudiante tiene un rol protagónico al capitalizar las tecnologías en su beneficio, lo cual deriva en el fortalecimiento de su capacidad de trabajar colaborativamente y su proactividad expresada a través de su liderazgo en el desarrollo de las tareas encomendadas y el ambiente de trabajo que desarrolla con su entorno (García-Pérez, Herrera-Rodríguez, García-Valero y Guevara Fernández, 2016); ello le permitirá a futuro adaptarse a las necesidades y situaciones que le deparen su diario accionar (Vinagre, 2014), e interculturalmente hacia entornos diversos (Paris, Mas y Torrelles, 2016), en los que se encuentre desempeñando el accionar profesional futuro.

El empleo de los ambientes virtuales, redes sociales, entre otras ayudas tecnológicas (García, Domínguez y Spitcich, 2017) potencia el desarrollo y fortalecimiento de una cultura colaborativa asertiva en el estudiantado (Olivares, 2015); sin embargo, el profesorado debe ponerse a tono con su propia competencia digital acortando con ello una brecha intergeneracional e intercultural (Rodríguez y Espinoza, 2017).

Es indudable que el profesor debe aprovechar las estrategias innovativas (Ferrer et al., 2016) para generar entre los estudiantes el espíritu competitivo para el afianzamiento del liderazgo proactivo y la cultura colaborativa en un conglomerado social, ambiental y económico para identificar los aspectos mejorables de su desempeño al momento de integrarse a él (Pachano, 2014).

Un colectivo de aula direccionado de forma adecuada por un docente con praxis innovadora (Ganga-Contreras, Navarrete, Alt y Alarcón, 2016), permitirá el empoderamiento del alumnado sin distinción de género (Toledo, Orús y Agudo, 2015)(Reche, Sachicola y Lucena, 2015), sobre todo en aquellos casos en los que existan actividades, acciones o responsabilidades tradicionalmente sesgadas a un género en específico (Sopo, Salazar, Guzmán y Vera, 2017)(Cuevas-López y Díaz-Rosas, 2015). 
Es destacable el trabajar en equipo conformado por estudiantes, profesores y autoridades (Escobar, Isabel y Guerra, 2017)(Cetzal y Marfileño, 2019), para que las competencias de cultura colaborativa y liderazgo se hagan de forma transversal (Delgado, Oliver y Rovira, 2015) al interior de la carrera de AGROPECUARIA, es decir que todos los actores del claustro universitario manejen y apliquen las mismas estrategias para beneficiar por igual a todos los futuros graduados.

\section{Conclusiones}

La valoración que el estudiantado de la carrera de AGROPECUARIA de la UCSG hace de su percepción sobre la importancia de desarrollar la cultura investigatíva es destacable. El liderazgo durante su formación educativa, se asocia a las capacidades del docente para motivar el desarrollo de dichas competencias. Es importante seguir trabajando en el fortalecimiento de éstas competencias e ir integrando otras que ayudarán a complementar las habilidades blandas en el colectivo del aula.

\section{Referencias}

Avalos, S., Dávila, C., y López-Walle, J. (2015). Climas motivacionales, liderazgo y cohesión grupal en contexto deportivo universitario. Educación Física y Ciencia, 17(1).

Borrasca, B. (2014). El aprendizaje colaborativo en la universidad: referentes y práctica. Revista de Docencia Universitaria, 12(4), 281302.

Cadavieco, J., Martínez, M. y Cabezas, I. (2016). Trabajo colaborativo en la educación superior: una competencia profesional para los futuros docentes. Educ. Soc. [Online], 37(135), 519-538.

Cetzal, R., y Marfileño, V. (2019). El liderazgo docente en la educación superior. Una propuesta considerando el MEI. Revista del Departamento de Formación y Actualización Académica de la UAA, (19), 7-9.

Cuevas-López, M., y Díaz-Rosas, F. (2015). Género y liderazgo en la universidad española. Un estudio sobre la brecha de género en la 
gestión universitaria. Education Policy Analysis Archives, 23 (106), $1-26$.

Delgado, A., Oliver, R. y Rovira, I. (2015). Las competencias de negociación, liderazgo y trabajo en equipo en un máster virtual. Coor. Tortoso, Álvarez y Pellín. XIII Jornadas de Redes de Investigación en Docencia Universitaria: Nuevas estrategias organizativas y metodológicas en la formción universitaria para responder a la necesidad de adaptación y cambio, 943-954. Alicant, España.

Domínguez, M., Ruiz, A. y Medina, A. (2017). Experiencias docentes y su proyección en la identidad profesional: el caso de las maestras de Santa Marta y su entorno. Rev. hist. edu. latinoam, 19(29), 111-134.

Escobar, J., Isabel, A., y Guerra, R. (2017). El liderazgo y la participación como factores clave para la gestión de la calidad. Caso de la Universidad Estatal de Bolívar. Cofin Habana, 11(2), 206-225.

Fernández, P. (2016). Acerca de los enfoques cuantitativo y cualitativo en la investigación educativa cubana actual. Atenas, 2(34), 1-15.

Ferrer, M., Giner, R., Cortés, D., Ríos, J., Ibáñez M., Serrano, A.,...y Santamarina, M. (2016). Flipped classroom en el aprendizaje multidisciplinar colaborativo en diferentes grados universitarios. Coord. por Tortoso, Grau y Álvarez, XIV Jornadas de Redes de Investigación en Docencia Universitaria: Investigación, innovación y enseñanza universitaria: enfoques pluridisciplinares, 2414-2424. Instituto de Ciencias de la Educación, Alicante, España.

Ganga-Contreras, F., Navarrete, E., Alt, C. y Alarcón, N. (2016). Percepción de los estilos de liderazgo: el caso de un campus universitario. Revista Dilemas Contemporáneos: Educación, Política y Valores, 1(36).

García, D., Domínguez, A. y Spitcich, S. (2017). Trabajo colaborativo para el desarrollo de prácticas innovadoras en la enseñanza de la física universitaria con el uso de tecnologías. Revista de Enseñanza de la Física, 29(1), 7-23. 
García-Pérez, Y., Herrera-Rodríguez, J., García-Valero, M. y Guevara-Fernández, G. (2015). El trabajo colaborativo y su influencia en el desarrollo de la cultura profesional docente. Gaceta Médica Espirituana [Online], 17(1).

Gómez, M. (2015). La dicotomía cualitativo-cuantitativo: posibilidades de integración y diseños mixtos. Campo Abierto. Revista de Educación, 1, 11-30.

Hernández, J., Tobón, S., y Vázquez, J. (2015). Estudio del liderazgo socioformativo mediante la cartografía conceptual. Revista Iberoamericana de Evaluación Educativa, 8(2), 105-128.

Magaña-Medina, D., Aguilar-Morales, N., y Avendaño-Rodríguez, K. (2016). Diferencias de género en capacidad de liderazgo percibido en estudiantes de pregrado. Opción: Revista de Ciencias Humanas y Sociales, (13), 173-195.

Medina, I., Trasfi, M., Armenteros, M. y Reyna, G. (2015). Relación Entre Cultura-Liderazgo: Evidencias en una Institución de Educación Superior. Revista Internacional y Finanzas, 8(4), 77-91.

Medina, M., Quintero, M. y Valdez, J. (2013). El enfoque mixto de investigación en los estudios fiscales. Tlatemoani, (13).

Moreno, J., Chiecher, A., y Paoloni, P. (2015). El ingreso en carreras de ingeniería: Facebook y su potencial para favorecer la integración a la cultura universitaria. Revista Argentina de Enseñanza de la Ingeniería, 4(9), 9-18.

Olivares, B. (2015). Implementación de la red social facebook como recurso didáctico en el aprendizaje colaborativo de estudiantes universitarios. Revista de estudios y experiencias en educación, 14(27), 121-136.

Pachano, N. (2014). Estrategias gerenciales para fortalecer el aprendizaje colaborativo de los estudiantes del programa de posgrado administración de la educación básica de la UNERMB, sede CABIMAS. Revista electrónica de Humanidades, Educación y Comunicación Social, 9(18), 79-101.

París, G., Mas, O. y Torrelles, C. (2016). La evaluación de la competencia 'trabajo en equipo' de los estudiantes universitarios. Revista d'Innovació Docent Universitària, 8, 86-97. 
Peralta, Y., Gandoy, F., Jara, C., y Pacenza, M. (2016). Liderazgo del docente y niveles de empoderamiento de los estudiantes en un seminario de prácticas comunitarias de una universidad pública argentina. Cuadernos de Administración, 31(54), 68-79.

Pérez, E., Medina, M. y Cachón, J. (2019). Perception of the Professional Competences of Last Year's Students of Pre-Primary Education and Primary Education Degrees and Students of Training Teachers Master. Journal of New Approaches in Educational Research, 8(1), 58-65.

Reche, M., Sachicola, A. y Lucena, M. (2015). Análisis del liderazgo femenino y poder académico en el contexto universitario español. European Scientific Journal, 11(2).

Rodríguez, R. y Espinoza, L. (2017). Trabajo colaborativo y estrategias de aprendizaje en entornos virtuales en jóvenes universitarios. Rev. Iberoam. Investig. Desarro. Educ, 7(14), 86109.

Romero, A. y Garay, U. (2018). Aprendizaje colaborativo a través de redes sociales en contextos universitarios. Revista Electrónica de Tecnología Educativa, (62), 62-72.

Roselli, N. 2016. El aprendizaje colaborativo: Bases teóricas y estrategias aplicables en la enseñanza universitaria. Propósitos y Representaciones, 4(1), 219-280.

Sopo, G., Salazar, M., Guzman, E. y Vera, L. (2017). Liderazgo como competencia emprendedora. Revista Espacios, 38(24).

Toledo, S., Orús, M. y Agudo, J. (2015). Análisis de la formación en liderazgo en las organizaciones educativas: un estudio en la Comunidad Autónoma de Aragón. Revista electrónica interuniversitaria de formación del profesorado, 18(3), 39-54.

Torres, A. y Vallejo, M. 2018. Organización y funcionamiento de los agrupamientos en Educación Superior: Concepciones de los estudiantes. Tendencias Pedagógicas, 32, 15-30.

UCSG, 2017. Rediseño curricular de la Carrera de Agropecuaria. Facultad de Educación Técnica para el Desarrollo. Universidad Católica de Santiago de Guayaquil. Proyecto curricular presentado 
al Consejo Universitario conforme los lineamientos dispuestos por el CES Consejo de Educación Superior.

Vinagre, M. 2014. El desarrollo de la competencia intercultural en los intercambios telecolaborativos. Revista de Educación a Distancia, 41. 